\title{
Extração de incisivo inferior: uma opção de tratamento
}

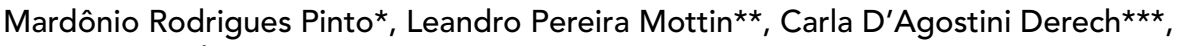

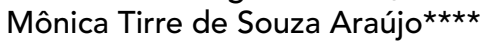

\section{Resumo}

A extração de incisivos inferiores pode ser considerada como opção de tratamento em casos de má oclusão Classe I com apinhamento inferior, principalmente quando é observada desproporção dentária entre a arcada superior e inferior; o que pode ser demonstrado através da análise de Bolton. Essa desproporção dentária, pode ocorrer devido à diminuição da largura dos dentes superiores ou aumento na largura dos dentes inferiores. Neste caso clínico, o tratamento de escolha, com extração de um incisivo inferior, proporcionou terapia rápida, com o mínimo de efeitos colaterais indesejáveis.

Palavras-chave: Incisivo inferior. Extração. Tratamento ortodôntico.

\section{INTRODUÇÃO}

Diversas opções de tratamento têm sido sugeridas para a resolução de casos com apinhamento ântero-inferior, tais como: movimento distal de dentes posteriores, expansão da arcada, projeção de incisivos, desgaste interproximal de dentes, extração de pré-molares e extração de incisivos ${ }^{28}$. Todavia, é necessário planejamento cuidadoso de cada caso, avaliando inclusive o potencial de recidiva para a opção escolhida.

A indicação de extração de dentes saudáveis como coadjuvante do tratamento ortodôntico já data de alguns anos, entretanto, a escolha do dente a ser extraído depende de algumas condições clínicas, como discrepância do arco dentário, desproporção entre dentes superiores e inferiores e perfil facial.

\section{REVISÃO DE LITERATURA}

Em 1757, Bourdet, um discípulo de Pierre Fauchard, já recomendava a extração de pré-molares para resolver problemas de apinhamento dentário ${ }^{7}$, entretanto, o primeiro relato de caso clínico com extração de incisivo inferior, como opção de tratamento, data de 1904, quando Jackson (1904, apud RIEDEL et al. ${ }^{21}$, 1991) publicou o tratamento de um paciente onde um incisivo tinha sido previamente removido e ele decidiu remover um segundo incisivo como opção de tratamento ${ }^{21}$.

Em um estudo de 20 anos pós-tratamento ortodôntico de um paciente que tinha perda congênita de dois incisivos inferiores, Schwarz (apud RIEDEL et al. ${ }^{21}, 1991$ ) mostrouse surpreso ao observar a boa estabilidade ${ }^{21}$. Em outro estudo de 10 anos pós-tratamento de

\footnotetext{
* Mestre em Ortodontia pela UFRJ, Doutorando em Ortodontia pela UFRJ, Coordenador e Professor do Curso de Especialização em Ortodontia pelo CEC/Universidade Estadual do Ceará.

** Mestre em Ortodontia pela UFRJ, Doutorando em Ortodontia pela UFRJ, Professor Adjunto da Disciplina de Ortodontia da Faculdade de Odontologia-UBRA/Torres.

*** Mestre em Ortodontia pela UFRJ, Doutorando em Ortodontia pela UFRJ.

**** Mestre, Doutora e Professora Adjunta da Disciplina de Ortodontia do Departamento de Odontopediatria e Ortodontia da Faculdade de Odontologia da UFRJ.
} 
casos tratados com extração de dois incisivos inferiores, Riedel ${ }^{20}$ observou que os arcos, nesses pacientes, apresentaram-se menos apinhados póstratamento quando comparados a casos similares tratados com extração de pré-molar. Joondeph e Riedel ${ }^{11}$ afirmam que a extração de dois incisivos inferiores deve satisfazer a necessidade de manter a forma do arco sem expandir a largura inter-caninos. O fato é que a extração de incisivo inferior como modalidade de tratamento sempre foi assunto polêmico. Apesar de muitos resultados favoráveis, o tratamento ortodôntico com extração de incisivo inferior ainda não é bem aceito. Alguns efeitos indesejáveis têm sido relatados como: overjet e overbite aumentados, recidiva do espaço da extração, oclusão insatisfatória, recorrência de apinhamento entre os incisivos remanescentes e perda de estética da papila gengival interdentária na região da extração $7,9,13,21,22,25,26,27$.

A literatura tem mostrado ${ }^{21}$ que tratamentos ortodônticos sem extração, em casos com grave apinhamento dentário ântero-inferior, normalmente apresentam recidiva do mesmo. Provavelmente isto se deve à forte tendência de diminuição do comprimento do arco inferior e no aumento da largura inter-caninos. Joondeph e Riede ${ }^{11}$ sugeriram que, em pacientes com grave apinhamento no arco inferior, a remoção de um ou mais incisivos inferiores fosse tomada como alternativa lógica para aumentar a estabilidade da região ântero-inferior sem a necessidade de contenção permanente. Em alguns casos, a extração de dois incisivos inferiores satisfaz os requisitos de manter a forma do arco sem alterar a largura inter-caninos ${ }^{9}$. Segundo esses autores ${ }^{11,21}$, dentre as indicações para a extração de incisivos inferiores estariam o apinhamento extremo e/ou protrusão, particularmente quando acompanhado de perda de tecido gengival ou perda da tábua óssea vestibular na região das raízes dos incisivos inferiores. Em um editorial publicado no American Journal of Orthodontics em 1963, Salzmann ${ }^{22}$ lembrou a filosofia de Angle sobre extração em Ortodontia, mostrando que o referido autor relutava em fazer extrações de incisivos e criticava o resultado oclusal final daqueles casos tratados com esse tipo de terapia.

Kokich e Shapiro ${ }^{13}$ estudaram a extração dos incisivos inferiores no tratamento ortodôntico e relataram que, em casos criteriosamente selecionados, onde é feito um planejamento cuidadoso, a discrepância oclusal resultante pode ser resolvida satisfatoriamente com uma mecânica relativamente simples, produzindo bons resultados. Riedel também sugeriu que a extração do incisivo inferior, para resolver apinhamento ântero-inferior, pode reduzir o tempo de tratamento ${ }^{11,21}$.

É necessário que, para cada caso onde pretenda-se solicitar a extração de um incisivo como parte da terapia ortodôntica, seja realizado um criterioso setup de diagnóstico ${ }^{6,17,21}$, como um importante auxiliar na confirmação do resultado esperado ${ }^{7}$. Quando corretamente confeccionado, o setup auxilia na tomada de decisões quanto às exodontias, aos desgastes interproximais, ao grau de ancoragem necessária, entre outros, indicando a melhor opção na decisão do planejamento de um tratamento, uma vez que permite a avaliação das limitações do caso antes de ser tratado ${ }^{3,6}$. O setup, juntamente com uma boa radiografia interproximal, orientará o ortodontista com relação à quantidade de esmalte mesiodistal que deverá ser removida dos incisivos superiores, caso necessário $^{13,14,24,26}$. Segundo Canut ${ }^{7}$, as indicações para a extração de incisivo inferior, resumem-se a quatro tipos de situações clínicas: anomalias de número dos dentes anteriores; anomalias de tamanho de dentes (possivel discrepância de Bolton ${ }^{4,5}$ ); erupção ectópica de incisivos; e má oclusão tipo Classe III moderada. É importante que o ortodontista, além do setup, tenha outros critérios que devem ser considerados, como: avaliação criteriosa da discrepância do arco dentário; verificação da largura inter-caninos e o efeito da extração de um incisivo sobre esta, já que a estabilidade do tratamento é melho, caso esta largura inicial seja mantida ${ }^{2,16,19}$; e obtenção de overbite e overjet adequados. 
Segundo Tuverson ${ }^{25,26}$, a extração de incisivos inferiores está contra-indicada nos seguintes casos: 1) quando o setup de diagnóstico demonstrar que um excessivo trespasse vertical (overbite) pode ser criado, não sendo possivel corrigí-lo através de movimentação dentária e/ou redução de esmalte mesio-distal dos dentes ântero-superiores; 2) pacientes com predisposição a doenças periodontais.

Este artigo teve como propósito relatar o caso clínico de uma paciente adulta com dois implantes de níquel-titânio na região dos incisivos centrais superiores, apinhamento ântero-inferior, relação molar de Classe I (Angle), padrão esquelético Classe I e overjet e overbite reduzidos, na qual a extração de um incisivo inferior foi o tratamento de escolha.

\section{APRESENTAÇÃO DO CASO}

Paciente do gênero feminino, 19 anos e 10 meses de idade, com bom estado geral de saúde, fonação e deglutição normais, simetria facial, terço inferior proporcional, bom selamento labial e perfil levemente convexo, com leve projeção do lábio inferior (Fig. 1). No exame intrabucal os arcos apresentavam-se parabólicos com prótese sobre implantes na região dos incisivos centrais superiores e considerável apinhamento na região ântero-inferior. A paciente era portadora de má oclusão Classe I de Angle com tendência à Classe III e a linha média inferior estava desviada aproximadamente $1 \mathrm{~mm}$ para o lado esquerdo. Apresentava mordida de topo anterior (overbite e overjet de $0 \mathrm{~mm}$ ) e discrepância dentária no arco inferior igual a $-6 \mathrm{~mm}$ (Fig. 3). No histórico médico não havia relato de nenhuma doença grave ou internação hospitalar, bem como de nenhum hábito bucal deletério, sendo sua respiração nasal.

$\mathrm{Na}$ radiografia panorâmica constatou-se a presença de todos os dentes permanentes com excessão dos incisivos centrais superiores, os quais haviam sido perdidos por traumatismo e substituídos por próteses sobre implantes. $\mathrm{Na}$ análise cefalométrica, o valor do ângulo ANB de $1^{\circ}$ evidenciou uma Classe I com tendência à Classe III esquelética o que é confirmado pelo SNA de $82^{\circ}$, frente a um SNB de $81^{\circ}$ e um SND de $78^{\circ}$. Os incisivos inferiores apresentavam uma inclinação axial vestibular levemente aumentada $\left(1 . \mathrm{NB}=26^{\circ}\right.$, $1-\mathrm{NB}=6 \mathrm{~mm})$. A análise de Bolton indicou excesso de tamanho dentário na arcada inferior de aproximadamente $4,4 \mathrm{~mm}$ de canino a canino. A avaliação periodontal indicou tecidos saudáveis e a paciente apresentava ótima higiene bucal.

\section{Objetivos do tratamento}

Os objetivos do tratamento eram: 1) corrigir pequenas alterações na arcada superior; 2) eliminar o apinhamento ântero-inferior; 3) promover estética satisfatória e ótimas condições de higiene bucal; 4) manter a harmonia facial e o tônus muscular; 5) compensar o excesso de massa dentária inferior com a extração de um incisivo inferior; e 6) restabelecer a função mastigatória normal com uma oclusão mutuamente protegida.

\section{Planejamento}

Uma consideração importante no plano de tratamento foi a harmonia facial apresentada pela paciente, principalmente com relação às alterações que poderiam advir no seu perfil. Após estudo cuidadoso do caso, foi executado um setup (Fig. 2) no intuito de servir como guia no planejamento e possibilitar uma visão mais próxima possível do resultado final do tratamento.
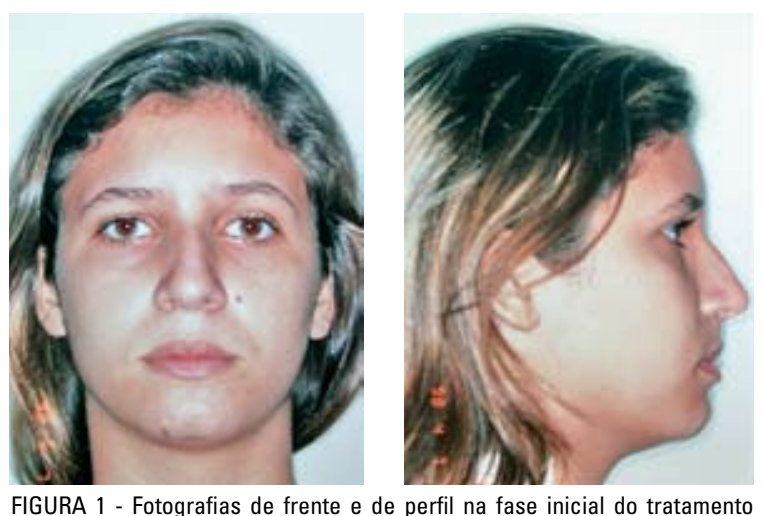

(observar a projeção do lábio inferior). 

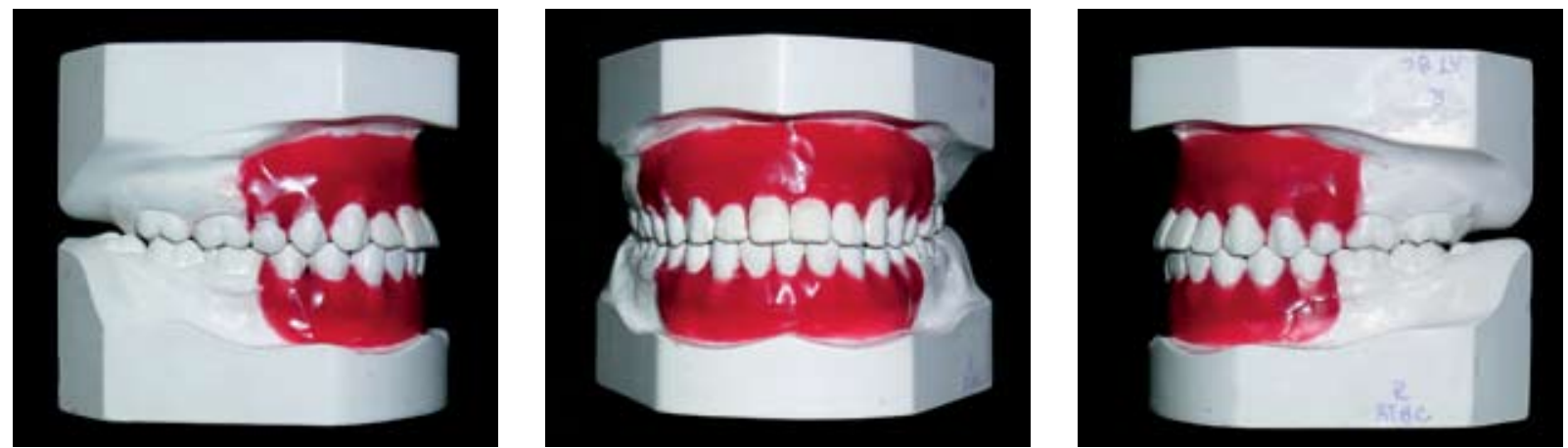

FIGURA 2 - Setup executado no intuito de servir como guia no planejamento do caso clínico.
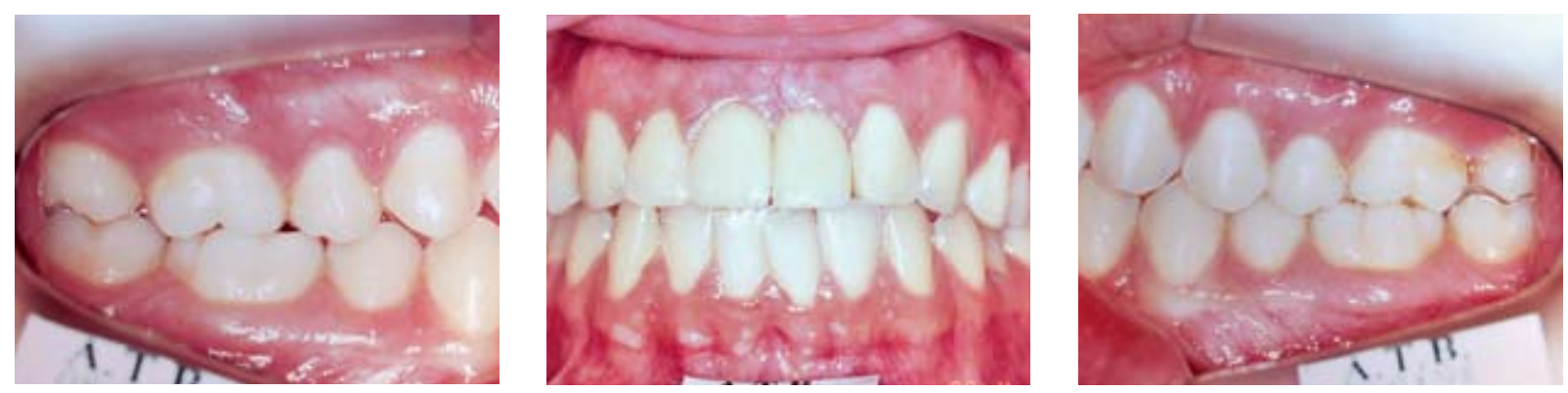

FIGURA 3 - Fotografias intrabucais: lateral direita, frente e lateral esquerda.
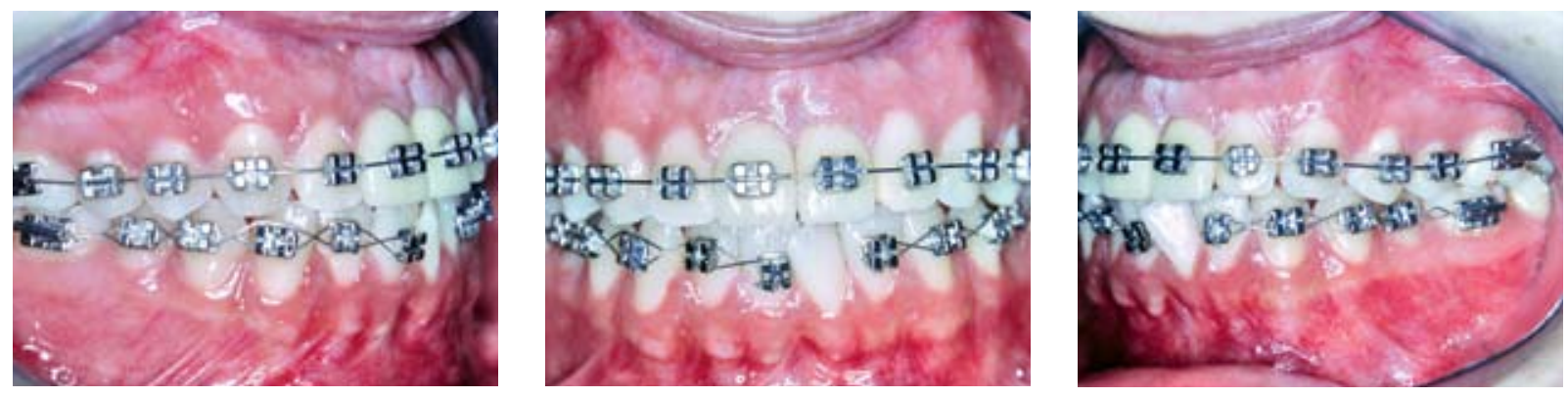

FIGURA 4 - Fotografias intrabucais mostrando o aparelho montado: lateral direita, frente e lateral esquerda.
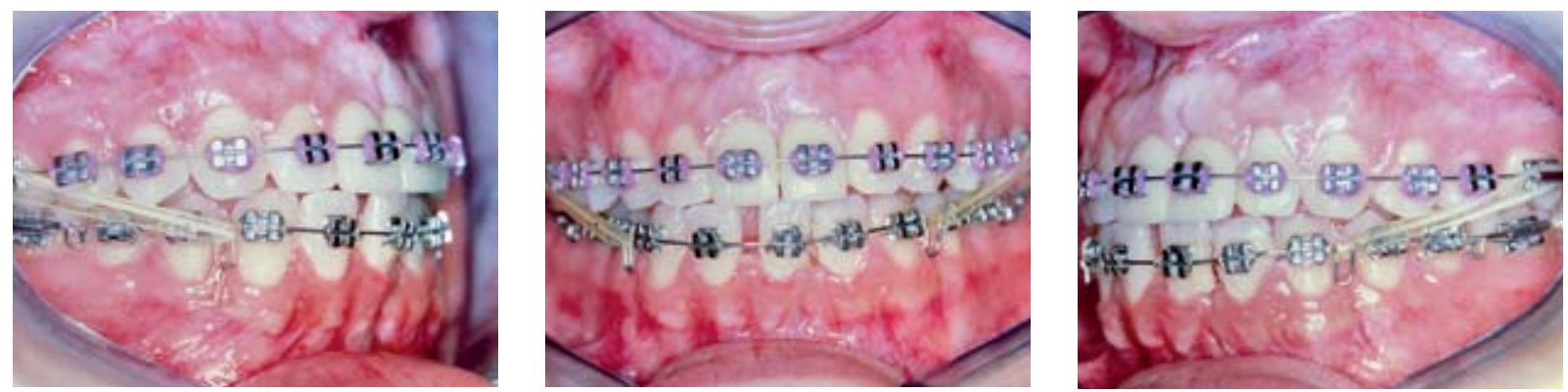

FIGURA 5 - Fotografias intrabucais mostrando o fechamento do espaço na região do incisivo extraído e a mecânica de Classe III com elásticos: lateral direita, frente e lateral esquerda. 
Baseado nos dados coletados optou-se por fazer o tratamento ortodôntico com extração do incisivo central inferior esquerdo devido à sua posição desfavorável $^{7}$ e à discrepância de Bolton evidenciada.

\section{Evolução do tratamento}

O tratamento foi iniciado com a montagem de aparelho ortodôntico fixo total tipo Edgewise .022" na arcada superior e inferior com todos os acessórios colados aos dentes, exceto o dente 31, o qual foi indicado para extração (Fig. 4). Após a retirada do referido dente, seguiu-se então ao alinhamento e nivelamento utilizando-se para isso a seguinte sequência de fios de secção redonda: níquel-titânio .012" e .014", e aço .016", .018" e .020". Dobras de compensação foram colocadas no arco inferior para prevenir inclinação excessiva de coroa para a região da extração. Foi utilizada cadeia elástica para fechamento do espaço ântero-inferior e após terminada a fase de alinhamento e ni- velamento foi colocado arco inferior .019" x .025" com alças de fechamento de espaço na mesial dos caninos inferiores, sendo ativado e auxiliado por mecânica de Classe III (elástico 5/16") para evitar mesialização dos segmentos posteriores (Fig. 5). Os ajustes no aparelho foram realizados em intervalos de 3 semanas. Finalizando o caso foram usados arcos .019" x .025" superior e inferior, coordenados com forma e torques ideais.

\section{Resultados}

O tratamento resultou em uma oclusão mutuamente protegida com Classe I molar e uma relação dos caninos estável. As fotografias finais mostraram boa harmonia facial e sorriso agradável (Fig. 6). A avaliação final das arcadas, mostrou que a discrepância dentária e o apinhamento do arco inferior haviam sido eliminados, e que o overbite e o overjet foram normalizados (Fig. 7). Os tecidos periodontais permaneceram saudáveis durante
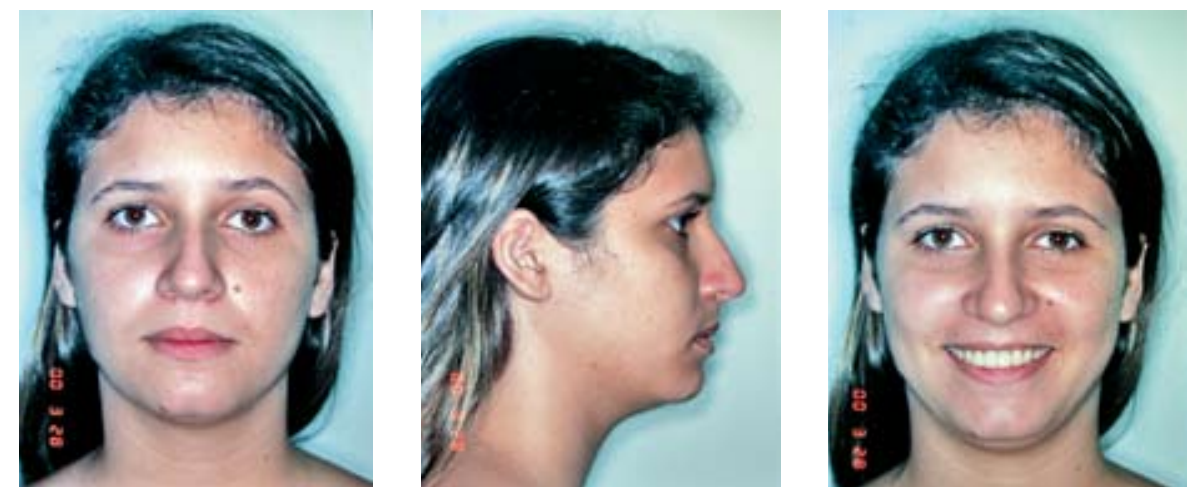

FIGURA 6 - Fotografias da paciente ao final do tratamento: frente, perfil e sorriso (observar a melhora na relação labial).
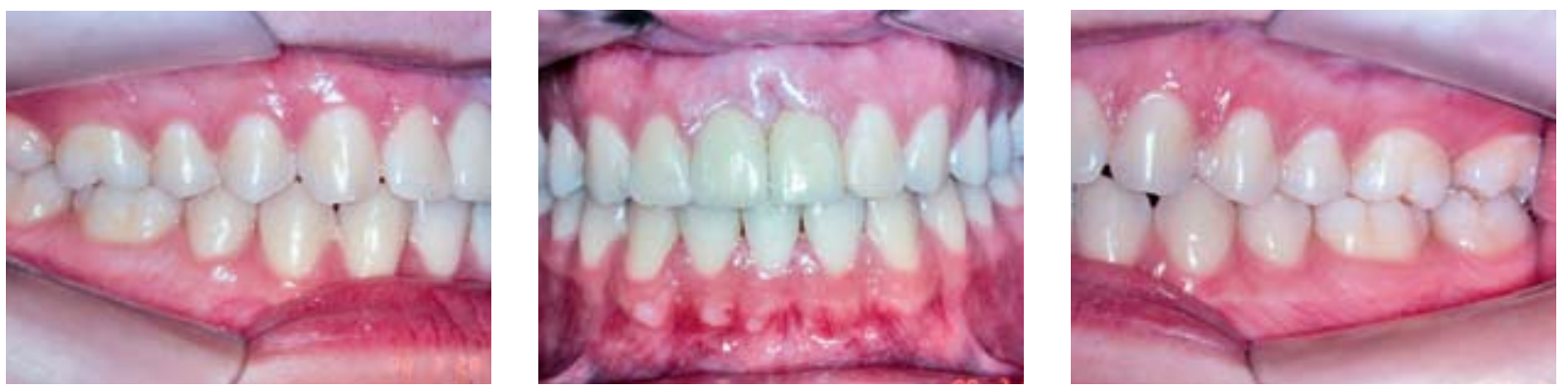

FIGURA 7 - Fotografias intrabucais ao final do tratamento: lateral direita, frente e lateral esquerda. 
todo o tratamento. A articulação têmporo-mandibular manteve-se com sua função normal durante todo o tempo de tratamento.

\section{Contenção}

O aparelho foi removido após 10 meses de tratamento e foi usado como contenção, no arco inferior, fio coaxial .0195" colado na lingual dos dentes ântero-inferiores de canino a canino e, no arco superior, placa de Hawley; com instrução para uso $24 \mathrm{~h}$ por dia, sendo retirada apenas no horário das refeições e na higiene bucal. Devido à probabilidade de impacção e falta de espaço, foram solicitadas as extrações dos terceiros molares. A paciente foi vista em intervalos de 1, 3 e 6 meses para avaliar a estabilidade da oclusão e o uso da contenção superior. Após um ano de contenção, foi solicitada a utilização da placa superior apenas no período noturno, pois a oclusão apresentava-se bem estabelecida. Dois anos após removido o aparelho fixo a paciente foi liberada do uso da placa de Hawley e a barra inferior foi removida, dando-se continuidade às visitas semestrais.

\section{DISCUSSÃO}

Nesse caso clínico, a decisão na escolha do tratamento com extração de um incisivo inferior, baseou-se nos trabalhos encontrados na literatu$\mathrm{ra}^{1-28}$. Em um artigo publicado em 1977, Bahreman ${ }^{1}$ relatou as indicações, contra-indicações e as vantagens da terapia ortodôntica com extração de um incisivo inferior. Entre as indicações encontravam-se as más oclusões Classe I com apinhamento ântero-inferior, onde a extração de quatro pré-molares prejudicaria o perfil facial ${ }^{19}$, o que foi observado no referido caso. Como vantagens Bahreman citou: 1) é consumido menos tempo de tratamento; 2) a mecanoterapia é usualmente simplificada; 3) a largura inter-caninos é mantida; 4) o período de contenção é reduzido; 5) geralmente não há problema de ancoragem. Em acordo com esse autor, o caso clínico aqui publicado mostrou um comportamento similar. O tempo de tratamento foi de 10 meses, a mecânica utilizada foi relativamente simples e o período de contenção de 2 anos, concordando com Bahreman ${ }^{1}$ e Braga et al. ${ }^{6}$

A paciente do caso relatado apresentou-se para tratamento com dois implantes de titânio na região de incisivos centrais superiores, o que a princípio limitava a movimentação na arcada superior. Apesar desta arcada apresentar-se relativamente harmoniosa, as próteses sobre implantes mostravam desproporção com os demais dentes (os incisivos tinham largura mésio-distal reduzida), o que colaborava com a discrepância quando da realização da análise de Bolton. Devido à recusa da paciente em retratar a região protética, optou-se pela extração do incisivo central inferior esquerdo, pois o mesmo apresentava-se mais desalinhado e já com comprometimento periodontal, tornando-o dessa forma o dente de escolha, segundo Canut ${ }^{7}$. Sendo assim, foi realizado um setup para auxiliar na confirmação da terapia escolhida. Uma segunda opção seria a realização de desgastes interproximais para compensar a discrepância encontrada; como proposto por Sheridan, Hastings ${ }^{24}$ e Tuverson ${ }^{25}$, entretanto, além da discrepância dentária de $-6 \mathrm{~mm}$, havia a necessidade de recolocação dos incisivos inferiores mais para lingual, impossibilitando assim essa alternativa de tratamento, pois a quantidade de esmalte a ser desgastado seria muito grande. Além disso, questões relativas à espessura do esmalte dos incisivos inferiores e o possível dano causado pelos desgastes tem sido levantadas ${ }^{13,14}$, bem como da reabsorção da crista óssea e o reduzido espaço para a papila interdentária ${ }^{13,27,28}$. Segundo Gilmore e Little ${ }^{10}$, o desgaste interproximal na coroa dos incisivos não garante melhor estabilidade pós-tratamento.

As opções de tratamento na mandíbula são mais limitadas que na maxila, devido, entre outras coisas, à ausência de sutura e ao osso cortical limitando a possibilidade de expansão com finalidade de conseguir espaços para corrigir apinhamento inferior ${ }^{27}$. Quando realizada, a mesma pode comprometer a integridade periodontal, com o aparecimento de áreas de retração gengival. 
Além de a preservação da largura inter-caninos ser de fundamental importância ${ }^{2}, 16,19$, sendo geralmente conseguida quando realizada a extração de um incisivo inferior para corrigir casos com apinhamento ${ }^{12}$.

A técnica utilizada no tratamento foi a Edgewise e, durante o fechamento de espaço, foi empregada força leve, mantendo-se sempre o cuidado em preservar o paralelismo radicular dos incisivos inferiores, o que era acompanhado através de radiografias periapicais, visto que, se o caso fosse finalizado com raízes divergentes, poderiam surgir espaços pós-tratamento na região da extração ${ }^{25}$.

A seleção desse caso, com moderado apinhamento ântero-inferior, nos possibilitou usar todo o espaço da extração a favor da correção da discrepância dentária, do overjet e do overbite, bem como reduzir o risco de perda da papila gengival, o que ocorre freqüentemente em adultos que apresentam severo apinhamento com comprometimento do tecido ósseo. Esses pacientes, quando submetidos a tratamento com extração de um incisivo, poderão ter resultados esteticamente insatisfatórios. Outro fator importante considerado foi o formato dos incisivos inferiores, os quais apresentavam forma mais retangular que triangular; pois quanto menos triangular, menor o risco de perda da papila gengival e do aparecimento de espaços nessa região. Quando diante de incisivos de formato triangular, é preferível direcionar o caso para tratamento com desgastes interproximais; caso não seja possível, a indicação recairá na extração de pré-molares a fim de manter a integridade da papila interdentária ${ }^{9}$. Infelizmente, o risco de uma retração gengival na região da extração existe, principalmente se o tecido mole dessa área for delgado. Para evitar o efeito desagradável de uma retração, o caso aqui relatado foi conduzido com a utilização de forças leves.

O tempo de tratamento desse caso clínico (10 meses) pode ser considerado relativamente curto se comparado com tratamentos em que são extraídos 4 pré-molares, encontrando-se abaixo da média quando comparado a casos na literatura usando a mesma terapia ${ }^{6,8,13,14,15,17,27}$.

Ao final do tratamento, foram verificados os movimentos de excursão lateral e anterior, observando-se a desoclusão posterior em ambos; procedendo-se então à retirada do aparelho. Após a remoção do mesmo, foi colocada contenção superior móvel tipo Hawley e contenção fixa inferior com fio coaxial .0195" colado de canino a canino para prevenir rotações e abertura de espaços, como no estudo de Zachrisson ${ }^{9}$. A paciente foi liberada do uso de contenção dois anos pós tratamento, devido à boa oclusão apresentada. Alguns estudos ${ }^{7,14,21}$ têm investigado a estabilidade pós-tratamento de casos tratados com extração de incisivo inferior. Riedel, Little e Buy ${ }^{21}$ avaliaram registros de pacientes 10 anos pós-contenção, tratados com extração de um incisivo e compararam com pacientes tratados com extração de pré-molar. Eles encontraram percentual maior de estabilidade naqueles casos tratados com extração de incisivos, o que concorda com o estudo realizado por Canut ${ }^{7}$.

Certamente a extração de incisivos inferiores tem sua indicação limitada, entretanto, quando criteriosamente planejada, poderá produzir ótimos resultados com estabilidade duradoura.

\section{CONCLUSÃO}

O tratamento ortodôntico de pacientes adultos com extração de um incisivo inferior pode ser viável em casos de reduzido overjet, reduzido overbite, discrepância de Bolton e leve tendência de relação molar de Classe III. No caso mostrado a oclusão e estética final foram satisfatórias, entretanto a construção do setup foi de fundamental importância como auxiliar no diagnóstico e planejamento do caso, a forma retangular dos incisivos inferiores e a utilização de forças leves e torques adequados contribuiram para um adequado posicionamento dos incisivos remanecentes com um mínimo de reabsorção dentária e para a preservação da papila interdentária na região da extração.

Enviado em: Fevereiro de 2004 Revisado e aceito: Julho de 2004 


\title{
Mandibular incisor extraction: a treatment option
}

\begin{abstract}
Extraction of lower incisors can be considered a treatment option in cases of Class I malocclusions with mandibular crowding, specially when a tooth-size discrepancy is observed between lower and upper arch; that can be demonstrated through Bolton analysis. This dental discrepancy is normally due to upper teeth's decrease in width and lower teeth's increase in width. In this clinical case, the treatment with lower incisor extraction, provided a quick therapy, with minimum undesirable effects.
\end{abstract}

Key words: Lower Incisor. Extraction. Orthodontic treatment.

\section{REFERÊNCIAS}

1. BAHREMAN, A. A. Lower incisor extraction in orthodontic treatment. Am J Orthod, St. Louis, v. 72, p. 560-567, 1977.

2. BLAKE, M.; BIBBY, K. Retention and stability: a review of the literature. Am J Orthod Dentofacial Orthop, St Louis, v. 114, p. 299-306, 1998.

3. BOLOGNESE, A. M. et al. Set-up: uma técnica de confecção. Rev SBO, Rio de Janeiro, v. 2, n. 8, p. 245-249, 1995

4. BOLTON, W. A. Disharmony in tooth size and its relation to the analysis and treatment of malocclusion. Angle Orthod, Appleton, v. 28, p. $113-130,1958$

5. BOLTON, W. A. The Clinical application of tooth-size analysis. Am J Orthod, St. Louis, v. 48, p. 504-529, 1962.

6. BRAGA, C. P. et al. "Set up" como auxiliar no diagnóstico da extração de incisivos inferiores. Ortodontia Gaúcha, Porto Alegre, v. 6, n. 2, p. 167-174, jul./dez. 2002.

7. CANUT, J. A. Mandibular incisor extraction: indication and longterm evaluation. Eur J Orthod, London, v. 18, p. 485-489, 1996.

8. CURIEL, P.; SANTORO, M. Treatment of patient with a crowed Class I malocclusion and a congenitally missing mandibular incisor. Am J Orthod Dentofacial Orthop, St Louis, v. 122, p. 661-665, 2002.

9. FAEROVIG, E.; ZACHRISSON, B. U. Effects of mandibular incisor extraction on anterior occlusion in adults with Class III malocclusion and reduced overbite. Am J Orthod Dentofacial Orthop, St. Louis, v. 115, p. 113-124, 1999.

10. GILMORE, C. A.; LITTLE, R. M. Mandibular incisor dimensions and crowding. Am J Orthod, St. Louis, v. 85, p. 493-463, 1984.

11. JOONDEPH, D. R.; RIEDEL, R. A. Retention. In: GRABER, T. M.; SWAIN, B. F. (Ed.). Orthodontics: current principles and techniques. St. Louis: C. V. Mosby, 1985. p. 857-898.

12. KLEIN, D. J. The mandibular central incisor, an extraction option. Am J Orthod Dentofacial Orthop, St. Louis, v. 111, p. 253-259, 1997.

13. KOKICH, V. G.; SHAPIRO, P. A. Lower incisor extraction in orthodontic treatment. Angle Orthod, Appleton, v. 54, p. 139-153, 1984.

14. KOKICH, V. O. JR. Treatment of a Class I malocclusion with a carious mandibular incisor and no Bolton discrepancy. Am J Orthod Dentofacial Orthop, St. Louis, v.118, p. 107-113, 2000.

15. MARCHIORO, E. M.; BELLATO A.; HANN, L. Extração de incisivo inferior na terapia ortodôntica: relato de caso clínico. Ortodontia Gaúcha, Porto Alegre, v. 6, n. 1, p. 17-22, jan./jun. 2002.

16. McCAULEY, D. R. The cuspid and its function in retention. Am J Orthod, St. Louis, v. 30, p. 196-205, 1944.
17. MILLER, R. J.; DUONG, T. T.; DERAKHSHAN, M. Lower incisor extraction treatment with the invisalign system. J Clin Orthod, Boulder, v. 36, no. 2, p. 95-102, Feb. 2002.

18. MOYERS, R. E. Handbook of Orthodontics. 4th ed. Chicago: Year book Medical, 1988. p. 230-231.

19. RIEDEL, R. A. A review of the retention problem. Angle Orthod, Appleton, v. 30, p. 179-194, 1960.

20. RIEDEL, R. A. Retention and relapse. J Clin Orthod, Boulder, v. 10, p. 454-472, 1976.

21. RIEDEL, R. A.; LITTLE, R. M.; BUY, T. D. Mandibular incisor extraction: post retention evaluation of stability and relapse. Angle Orthod, Appleton, v. 62, p. 103-116, 1991.

22. SALZMANN, J. A. Editorial: EH Angle on extraction in orthodontics. Am J Orthod, St. Louis, v. 49, no. 6, p. 464-466, 1963.

23. SHASHUA, D. Treatment of a Class III malocclusion with a missing mandibular incisor and severe crowding. Am J Orthod Dentofacial Orthop, St. Louis, v. 116, p. 661-666, 1999.

24. SHERIDAN, J. J.; HASTINGS, J. Air-rotor stripping and lower incisor extraction treatment. J Clin Orthod, Boulder, v. 26, no. 1, p. 18-22, Jan. 1992.

25. TUVERSON, D. L. Anterior interocclusal relations. Part I. Am J Orthod, St. Louis, v. 78, no. 4, p. 361-370, Oct. 1980.

26. TUVERSON, D. L. Anterior interocclusal relations. Part II. Am J Orthod, St. Louis, v. 78, no. 4, p. 371-393, Oct. 1980.

27. VALINOTI, J. R. Mandibular incisor extraction therapy. Am J Orthod Dentofacial Orthop, St. Louis, v. 105, p. 107-116, 1994.

28. ZACHRISSON, B. U. latrogenic damage in orthodontic treatment. J Clin Orthod, Boulder, v. 12, p. 112-113, 1978.
Endereço para correspondência

Mardônio Rodrigues Pinto

Rua Vicente Linhares, 1570, Apto 901

CEP: 60.135-270 - Fortaleza/CE

E-mail: mardoniopinto@ig.com.br 